\title{
Effect of Fiber Waviness on the Compressive Strength of Unidirectional Carbon Fiber Composites
}

\author{
Paul Davidson*, Anthony M. Waas ${ }^{\dagger}$ \\ Univeristy of Michigan, Ann Arbor, MI 48105, USA. Fellow, AIAA. \\ Chandra Sekar Yerramalli; \\ Karthick Chandraseker§, \\ Waseem Faidi \\ General Electric Global Research Center, Niskayuna, NY 12309, USA
}

\begin{abstract}
Compression strength experiments were conducted on carbon fiber unidirectional specimens with defects of varying aspect ratios and corresponding misalignment angles. The results showed a strong influence of misalignment angle in strength knockdown. A local-global modeling approach was used to simulate and predict kink banding, which was observed to be a strength limiting mechanism. The numerical model was also used to conduct a parametric study on the influence of misalignment angle and defect aspect ratio.
\end{abstract}

\section{Introduction}

The compression strength of a laminated composite structure is a design driver in many applications in the aerospace, automotive and wind turbine industries. Unintended and small misalignment in fiber angles can lead to a drastic knock down in compressive strength. ${ }^{1,2}$ Since manufacturing induced misalignment cannot be completely eliminated, it is important to understand the basic failure mechanisms induced by these defects and how these influence the compressive strength of composites. Compression failure in a laminated composite, in the presence of defects, is complex because there are multiple failure modes (kinking, ${ }^{1,3}$ splitting, ${ }^{2,3}$ de-lamination and buckling) that can occur, and in some cases these modes can interact, ${ }^{4,5}$ Each failure mode results in the lowering of compressive strength of the composite, but some failure modes are more catastrophic than others.

\footnotetext{
*Graduate Student Research Assistant, Department of Mechanical Engineering.

${ }^{\dagger}$ Felix Pawlowski Collegiate Professor,, Department of Aerospace Engineering.

${ }^{\ddagger}$ Senior Mechanical Engineer, Composites Design and Analysis Lab.

$\S$ Mechanical Engineer, Lifing Lab.

『Senior Mechanical Engineer, Non Destructive Testing Lab.
} 
Past studies on the effect of fiber waviness have examined the interaction of waviness with the reduction in the tangent stiffness of the matrix. ${ }^{2}$ Other studies by Mandell and Samborsky ${ }^{6}$ have looked at the effect of fiber waviness experimentally on the strength knockdowns. In this study we concentrate on failure due to fiber waviness in thick unidirectional carbon composites. The effects of a single type of defect on compression strength has been examined systematically by conducting coupon level experiments to obtain compressive response. A parametric micro-mechanical modeling approach is proposed to simulate and predict the observed experimental results, that shed new insights into compression response and failure. Thick composites, as will be explained later, have a non-uniform geometry which induces non-uniform stresses in the composite. The main cause of variation is the variation in spacing of fibers over the defect causing a change in volume fraction across the cross-section. In this study this variation is captured in the finite element formulation and a global-local approach is utilized to analyze and predict compression strength.

\section{Experiment}

\section{A. Fabrication \& Testing}

The first task of the study was to manufacture coupons with controlled defect geometry that reflects the actual defects in field. Field defects are categorized based on shape of the defect as being sinusoidal (generally low aspect ratio) or high aspect ratio. Sinusoidal defects conform to a sine profile, whereas high aspect ratio defects induce waviness due to the edge profile but have a flat peak.

Defects were introduced by inserting pre-cured resin blocks located at the center of the coupon with respect to length and height, $h_{d}$, measured from the bottom flat surface. The size of defect is defined by its aspect ratio $R_{d}=A_{d} / L_{d}$, which appears on the top surface as a bulge and is also defined by aspect ratio $R_{b}=A_{b} / L_{b}$, as shown in Figure 1 . Measurement of cured coupons reveal that $R_{d}>R_{b}$, which is due to the tendency of fibers to assume a minimum bending angle, causing the defect wavelength to increase as one moves outwards from the root of the defect. Due to this, the fiber volume fraction varies from near the defect to the outer edge. Post cure nominal volume fractions of the specimens was determined from microscopic image analysis of samples and found to be $V_{f}=0.434 \pm 0.027$, figure 2 . It was also noticed that cured samples showed distinct unidirectional layers of thickness $0.4907 \pm 0.1071$, as seen in figure 3. In addition, misalignment angle $(\alpha)$ of fiber above the defect, was also measured by pixel measurement of scanned cross-sections of the specimens. Table(1) provides the nominal dimensions of specimens used for the experiments, where specimens are classified based on defect location as: BD-bottom defect, MD-middle defect, TD-top defect.

Quasi-static compression tests were conducted on an MTS ${ }^{\circledR}$ high force servo-hydraulic test system with a Wyoming Combined Loading Compression (CLC) Test Fixture. The ends of each specimen were squared and assembled flush with the test fixture to avoid end effects. The CLC fixture is used to apply compressive load axially along the length of the specimen and to clamp the specimen on the end as shown in Figure 4. Tests were conducted at a crosshead displacement rate of $1 \mathrm{~mm} \cdot \mathrm{min}^{-1}$. For measurement of strains, a speckle pattern was applied on each specimen and high resolution images were taken using a digital camera at a framing rate of 1 frame per second. Strain fields were obtained by post processing the 
Table 1. Specimen type and dimensions.

\begin{tabular}{cccccc}
\hline $\begin{array}{c}\text { Class } \\
(-)\end{array}$ & $\begin{array}{c}H_{c} / H_{d} \\
(\mathrm{~mm})\end{array}$ & $\begin{array}{c}R_{d} \\
(\mathrm{deg})\end{array}$ & $\begin{array}{c}R_{b} \\
(\mathrm{~mm})\end{array}$ & $\begin{array}{c}\alpha \\
(\mathrm{mm})\end{array}$ & $L_{b}$ \\
\hline $\mathrm{BD}$ & 0.03 & $0.16 \sim 0.25$ & $0.014 \sim 0.24$ & $12.53 \sim 33.69$ & $44.0 \sim 154.5$ \\
$\mathrm{MD}$ & 0.5 & $0.041 \sim 0.068$ & $0.03 \sim 0.04$ & $18.05 \sim 19.29$ & $86.28 \sim 142.09$ \\
$\mathrm{TD}$ & 0.730 & 0.027 & 0.021 & 9.94 & 113.38 \\
\hline
\end{tabular}

images using Digital Image Correlation (DIC) software Aramis ${ }^{\circledR}$.

\section{B. Observations}

All specimens failed catastrophically characterized by the sudden and large drop in load and audible sound. It was also observed that in all cases the insert de-bonded first usually along the bottom of the defect. This caused the propagation of a de-lamination along the plane of insert thereby separating the wavy and straight part of the component. Though the de-lamination did not result in significant loss in load, it did cause global buckling in large aspect ratio defects. The failure mode causing a catastrophic load drop varied from in-plane kink band formation to out-of-plane kink banding* . Figure 5 depicts different failure modes, Out-of-plane Kinking (OK), and In-plane Kinking (IK) observed in experiments. Figure 6 shows typical force-displacement plots for IK failure. Figure 7, shows examples of failure of coupons due to IK . It is interesting to note that in the case of pure IK failure, as seen in higher $\alpha$ misalignments, even the matrix rich region undergoes bending due to kinking and but does not delaminate.

\section{Results}

The results of compression tests are shown in Figure(8) where experimental compressive strength $\left(X_{c}\right)$ normalized by the compressive strength of UD carbon $\left(X_{c}^{b}\right)$, calculated for $2^{\circ}$ imperfection using Budiansky's model ${ }^{11}\left(X_{c}^{b}=G_{m} /\left(1+\frac{\bar{\phi}}{\gamma_{m}}\right)\right)$, is plotted against $R_{d}$ in Figure(8-a) and $\alpha$ Figure(8-b). One outlier in the data corresponds to $B D 3$ which, due to it large aspect ratio and thin wavy section, showed global buckling and delamination. The results seem to be insensitive to $R_{d}$ as is seen from figure(8-a), which is because the aspect ratio of defect $R_{d}$ is not a true measurement of fiber misalignment. Though the defect causes misalignment, the profile of the fiber over the defect will not conform to the defect profile, but will tend to a profile offering the least angle of bend. As is seen in figure(8-b), the compressive strength shows much better spread with respect to $\alpha$. Coupons with small $\left(\sim<10^{\circ}\right)$ misalignments, kink out of plane (in $x-z$ plane) because the crosssection is constraint free in the $z$ direction but clamped in the $y$ direction. By comparing the failure modes observed with the corresponding misalignment angle, a transition in the failure mechanism from out-of-plane kinking to in-plane kinking is observed. In general, the compressive strength reduced with increasing misalignment angle.

\footnotetext{
${ }^{*}$ Prior studies by Yerramalli and Waas,${ }^{7}$ have alluded to the 3 -D nature of kink banding
} 


\section{Numerical analysis}

\section{A. Modeling}

An examination of the coupon geometry indicated that there are six parameters that control the fiber waviness as shown in Figure $9 ; \alpha, \phi, L_{d}, L_{b}, A_{d}, A_{b}$. To model the fiber profile, a cubic spline is defined as as a function of defect height and length:

$$
P_{X}(x)=\begin{array}{ccc}
\frac{3}{4} \frac{A_{d}}{h^{2}}\left(x+L_{d}\right)^{2}-\frac{1}{4} \frac{A_{d}}{h^{3}}\left(x+L_{d}\right)^{3} & -L_{d} \leq x \leq-h \\
\frac{A_{d}}{2}+\frac{3}{4} \frac{A_{d}}{h}\left(x+L_{d} / 2\right)-\frac{1}{4} \frac{A_{d}}{h^{3}}\left(x+L_{d} / 2\right)^{3} & \forall & -h \leq x \leq 0 \\
A_{d}-\frac{3}{4} \frac{A_{d}}{h^{2}}(x)^{2}+\frac{1}{4} \frac{A_{d}}{h^{3}}(x)^{3} & \forall & 0 \leq x \leq h \\
\frac{A_{d}}{2}-\frac{3}{4} \frac{A_{d}}{h}\left(x-L_{d} / 2\right)+\frac{1}{4} \frac{A_{d}}{h^{3}}\left(x-L_{d} / 2\right)^{3} & \forall & h \leq x \leq L_{d}
\end{array}
$$

where, $h=L_{d} / 2$ and the inflection point of curvature is at $\left( \pm L_{d} / 2, A_{d} / 2\right)$. The maximum angle of misalignment is given by:

$$
\tan (\alpha)=\frac{3}{2} \frac{A_{d}}{L_{d}}
$$

It should be noted that the traditional non-dimensional parameter $R_{d}$ does not give the maximum misalignment angle which is at the inflection point of the curvature over the defect. This is especially true for high aspect ratios. Using the above equations we can now define the fiber profile over the defect, however, as we move from the defect to the outer edges, the wavelength increases. To capture this, a propagation angle $\phi$ is used, which is defined as:

$$
\tan (\phi)=\frac{\left(H_{c}-H_{d}\right)}{\left(L_{b}-L_{d}\right)}
$$

Propagation angle will be a function of fiber bending stiffness, coupon thickness, location of defect, curing pressure and matrix properties. Predicting the propagation angle (due to manufacturing) is beyond the scope of this study, hence, an empirical value measured from the coupons were used. For the bottom defect, it was found that $\phi=21.707 \pm 2.71 \mathrm{deg}$, and for the middle defect and top defect $\phi \approx 12 \mathrm{deg}, \phi \approx 10 \mathrm{deg}$ were obtained, respectively. For the finite element based micro-mechanical model, the same cubic spline and propagation angle is followed with an additional constraint that the diameter of fiber is kept constant. The additional constraint ensures that change in volume fraction due to non-normal propagation, as seen in coupons, is captured. This is important because the location of shear stress concentration will also depend on the fiber volume fraction change along the wave.

\section{B. 2D Micromechanics based kink-band analysis}

For in-plane kinking, a micro-mechanical model of the composite ${ }^{8}$ with alternating layers of fiber \& matrix is used. Fibers are modeled as homogeneous orthotropic continua and the matrix is modeled as an isotropic material. In the past, Yerramalli et al, ${ }^{8}$ Pimenta et a ${ }^{10}$ have used a similar approach to obtain the kinking strength of a composite. Yerramalli et $\mathrm{al}^{8}$ have also shown scaling studies where in lieu of conducting a full scale micro-mechanics 
model, which is computationally expensive, a scaled down coupon can be used. For this study we follow a global-local approach as defined in the work by Ahn \& Waas. ${ }^{12}$

The global macro-mechanical model is a layered full scale homogenized model, with each layer modeled as homogeneous orthotropic material and the local material orientation is changed continuously based on the profile of the layer, as shown in Figure 10. Thickness of each layer is kept as $0.5 \mathrm{~mm}$ (mean thickness measured from coupons is $0.491 \pm 0.107$ ). The local micro-mechanical model is a sub-region of the global model but with fiber and matrix modeled explicitly. The fiber is modeled orthotropic, elastic, while the matrix is modeled as an elastic-plastic J2, incremental theory of plasticity solid. Here again, the material orientation of the fiber follows the fiber profile.

Lamina properties were obtained from the Concentric Cylinder Model (CCM) using fiber (listed in table 2) and matrix material properties obtained from $\mathrm{Ng}$ et al. ${ }^{13}$ The matrix non-linear in-situ equivalent stress-strain properties were taken from $\mathrm{Ng}$ et al. ${ }^{13}$

Table 2. Fiber Properties

\begin{tabular}{ccccccccc}
\hline$E_{11}$ & $E_{22}$ & $E_{33}$ & $G_{12}$ & $G_{13}$ & $G_{23}$ & $\nu_{12}$ & $\nu_{13}$ & $\nu_{23}$ \\
$(G P a)$ & $(G P a)$ & $(G P a)$ & $(G P a)$ & $(G P a)$ & $(G P a)$ & $(-)$ & $(-)$ & $(-)$ \\
\hline 276 & 20.5 & 20.5 & 32.8 & 32.8 & 7.6 & 0.28 & 0.28 & 0.28 \\
\hline
\end{tabular}

The local-global approach implemented here is a three step procedure using a macromechanical model and a micro-mechanical model as depicted in Figure 11. Step 1, is a linear elastic step where a known displacement is applied in the axial $(x)$ direction and the displacements $\left(\Delta_{x, y}\right)$ at nodes corresponding to local boundary $\left(A^{\prime}-B^{\prime}-C^{\prime}-D^{\prime}\right)$ are obtained. These displacements $\Delta_{x, y}$ are then applied on the local micro-mechanics model boundary and a linear elastic analysis is performed in step 2, henceforth called "Local Liner Model". In step 3, the reaction forces obtained $\left(R_{x, y}\right)$ in step 2 are applied on boundaries $\left(A^{\prime}-B^{\prime}\right) \&\left(C^{\prime}-D^{\prime}\right)$ along with $\Delta_{x, y}$ on $\left(A^{\prime}-D^{\prime}\right) \&\left(B^{\prime}-C^{\prime}\right)$ and a non-linear Riks response analysis is conducted ("Local Non-linear Model"). By applying reaction forces instead of displacement, the top and bottom boundaries are kept free of constraints, thereby allowing for kink band formation. The application of reaction forces is also important to ensure that the local model follows the global deformation profile in the elastic regimen.

The axial reaction force $R_{x}$ obtained from steps $1 \& 2$ provide the linear relation between local and global as a simple ratio $\hat{R}=\left(R_{x}^{\text {global }} / R_{x}^{\text {local }}\right)$. Now, from step 3 , we get the microkink initiation load $\left(R_{k}^{\text {local }}\right)$ which will correspond to the global kink strength $\sigma_{k}$ as ;

$$
\sigma_{k}=\left(R_{k}^{\text {global }}\right) / A=\left(\hat{R} R_{k}^{\text {local }}\right) / A
$$

To determine the appropriate size of the local-region, a scaling study was conducted where the size was scaled by the number of fibers in the thickness direction as shown in Figure 12, while keeping the aspect ratio $\left(\frac{A^{\prime}-B^{\prime}}{A^{\prime}-D^{\prime}}\right)$ fixed. Figure 12 also shows the plot of normalized $X_{c}$ as the number of fibers are increased. Strength values converge with increasing number of fibers and the number of fibers for subsequent simulations is taken as 150 . 


\section{Results}

Simulations were conducted for defect angles, $\alpha=30^{\circ}, 20^{\circ}, 15^{\circ}, 10^{\circ}, 5^{\circ}$ with $H_{d}=1,2,3 \mathrm{~mm}$ to examine the trend in kinking strength. Figure 14 shows the results of the parametric study. With an increase in both misalignment angle and defect height, a decrease in kink strength is predicted. Defect height is seen to have less influence on the kink strength with increasing misalignment angle. The results were compared with experimental data for defects of $H_{d}=1 \mathrm{~mm}$ as shown in Figure 15. Simulations were conducted on specimens corresponding to mean and maximum fiber volume fractions estimated from image analyses. The simulation results are within the experimental scatter as shown in the figure.

\section{Conclusion}

The experimental studies conducted thus far have shown that there is a significant knockdown in compressive strength of unidirectional composite laminates. The problem studied here is complicated due to the various geometric and material variability introduced by defects. Modeling of the problem using a global-local approach has been validated and is shown to provide a very good prediction for the compressive strength. By using the localglobal method, it is possible to model the actual problem without significant computational cost as compared to a full scale simulation or a scaled down model.

\section{Acknowledgement}

This material is based upon work supported by the Department of Energy under Award DE-EE0001367. The authors are grateful for this support.

\section{References}

${ }^{1}$ B. Budiansky, N.A. Fleck, and J.C. Amazigo. On kink-band propagation in fiber composites. Journal of the Mechanics and Physics of Solids, 46(9):1637 - 1653, 1998.

${ }^{2}$ S.H. Lee and A.M. Waas. Compressive response and failure of fiber reinforced unidirectional composites. International Journal of Fracture, 100(3):275-306, 1999.

${ }^{3}$ S. H. Lee, Chandra S. Yerramalli, and Anthony M. Waas. Compressive splitting response of glass-fiber reinforced unidirectional composites. Composites Science and Technology, 60(16):2957 - 2966, 2000.

${ }^{4}$ P. Prabhakar and A.M Waas. Investigation of Compression Strength and Post-Buckling failure in Multi-Directional laminated composites, International Conference on Composites for 21st Century: Current \& Future Trends, Indian Institute of Science, Bangalore, India, January 2011.

${ }^{5}$ P. Prabhakar and A.M Waas. Interaction between buckling and delamination in compressive failure of fiber reinforced composites, Advanced Topics in Aerospace Materials and Structures III, ASME International Mechanical Engineering Congress 83 Exposition, Vancouver, British Columbia, Canada, November 2010.

${ }^{6}$ J.F. Mandell, D.D. Samborsky, and L. Wang. Effects of fiber waviness on composites for wind turbine blades,. In Int. SAMPE symposium, 2003.

${ }^{7}$ C.S. Yerramalli and A.M. Waas. The Effect of Fiber Diameter on the Compressive Strength of Composites - A 3D Finite Element Based Study Computational Modeling in Engineering and Science, vol.6, no.1, pp.1-16, 2004

${ }^{8}$ C.S. Yerramalli, T. Miebach, K Chandraseker, and S.C Quek. Fiber waviness induced strength knockdowns in composite materials used in wind turbine blades. In EWEC 2010 Proceedings, 2010. 
${ }^{9}$ De Xie and Anthony M. Waas. Discrete cohesive zone model for mixed-mode fracture using finite element analysis. Engineering Fracture Mechanics, 73(13):1783 - 1796, 2006.

${ }^{10}$ S. Pimenta, R. Gutkin, S. T. Pinho, P. Robinson. A micromechanical model for kink-band formation: Part I Experimental study and numerical modelling. Composites Science and Technology 69 948955, 2009

${ }^{11}$ B. Budiansky, N.A. Fleck. Compressive failure of fibre composites. J Mech Phys Solids 1993;41:183-211

${ }^{12}$ J. Ahn, A.M.Waas, Stress Gradient Effects on Notched Composite Failure using a Local-Global Approach. 45th AIAA Structures, Structural Dynamics 83 Materials Conference 2004 AIAA-2004-1844

${ }^{13}$ W.H. Ng, A.G. Salvi, A.M. Waas, Characterization of the in-situ non-linear shear response of laminated fiber-reinforced composites. Composites Science and Technology 70 (2010) 11261134 


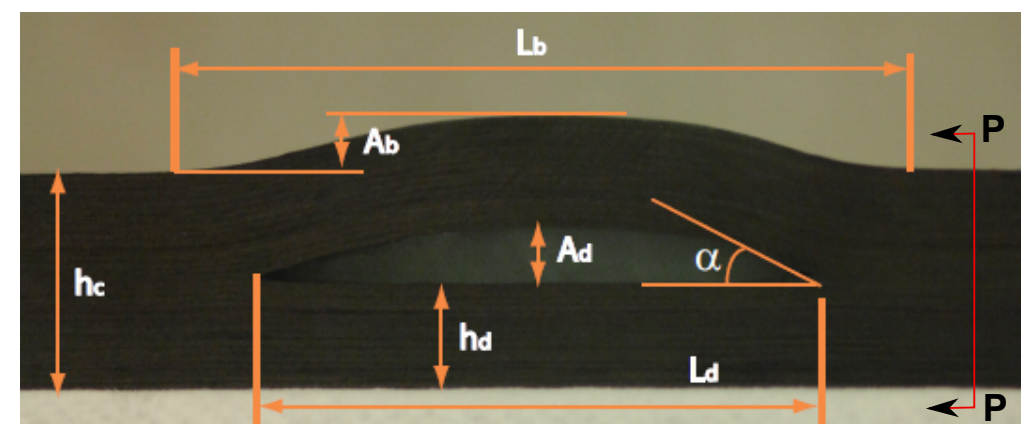

Figure 1. Dimensions of compression test coupons with defect inserts.
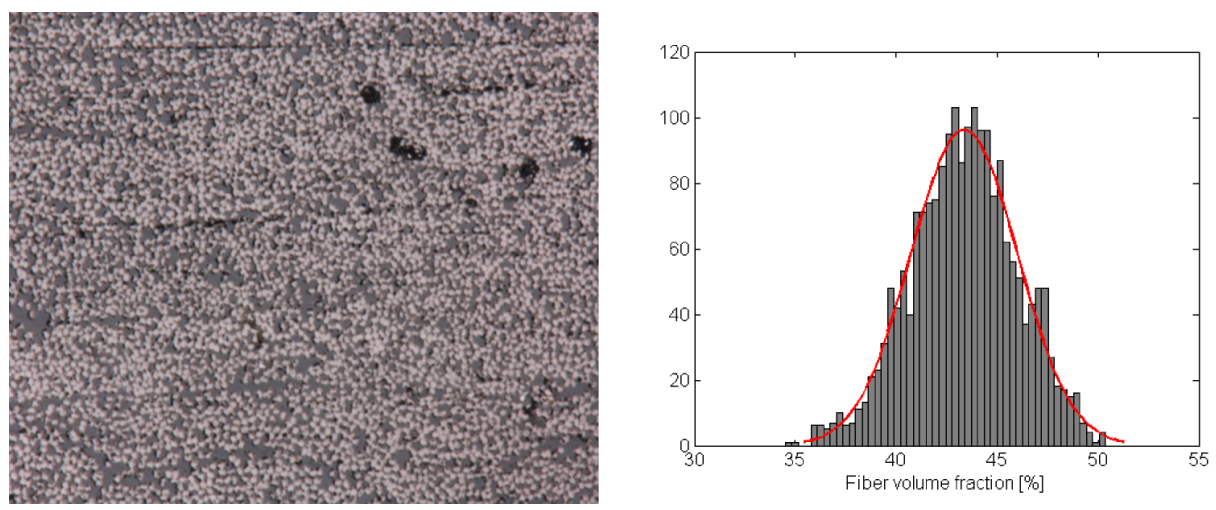

Figure 2. Microscopic image of composite cross-section (P-P in figure 1) and volume fraction PDF $(\mu=43.37 \%, \sigma=2.64 \%)$. 


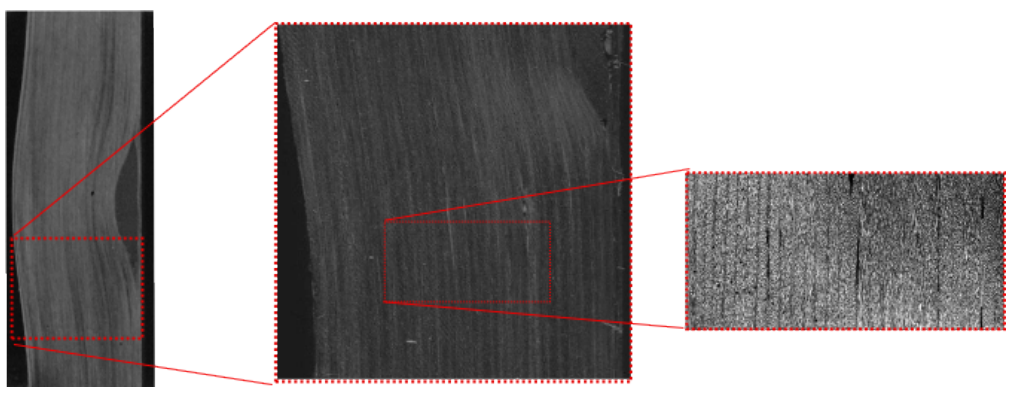

Figure 3. Microscopic image showing layers in UD coupon.

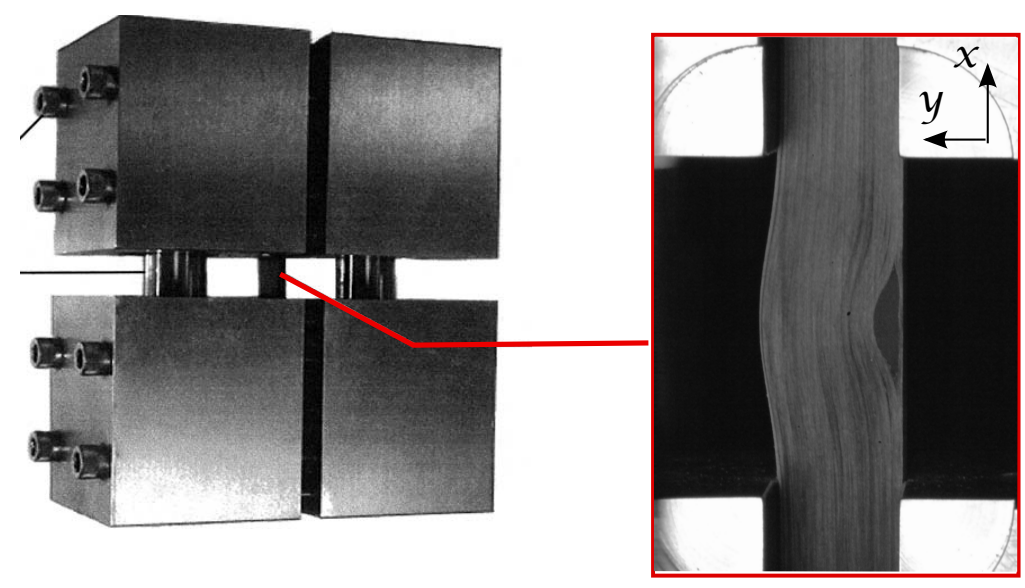

Figure 4. Wyoming CLC compression fixture.

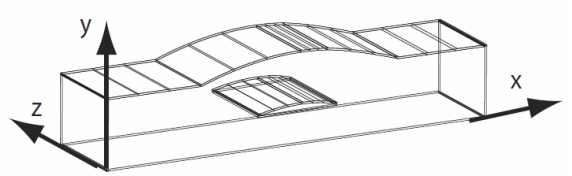

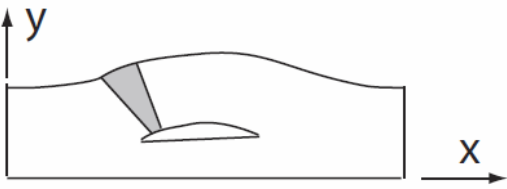

In-plane kink

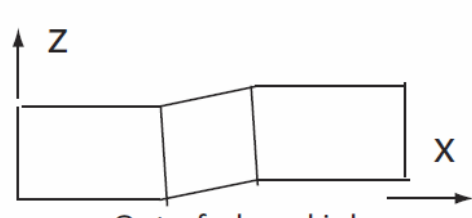

Out-of-plane kink

Figure 5. Failure modes seen in experiments. 

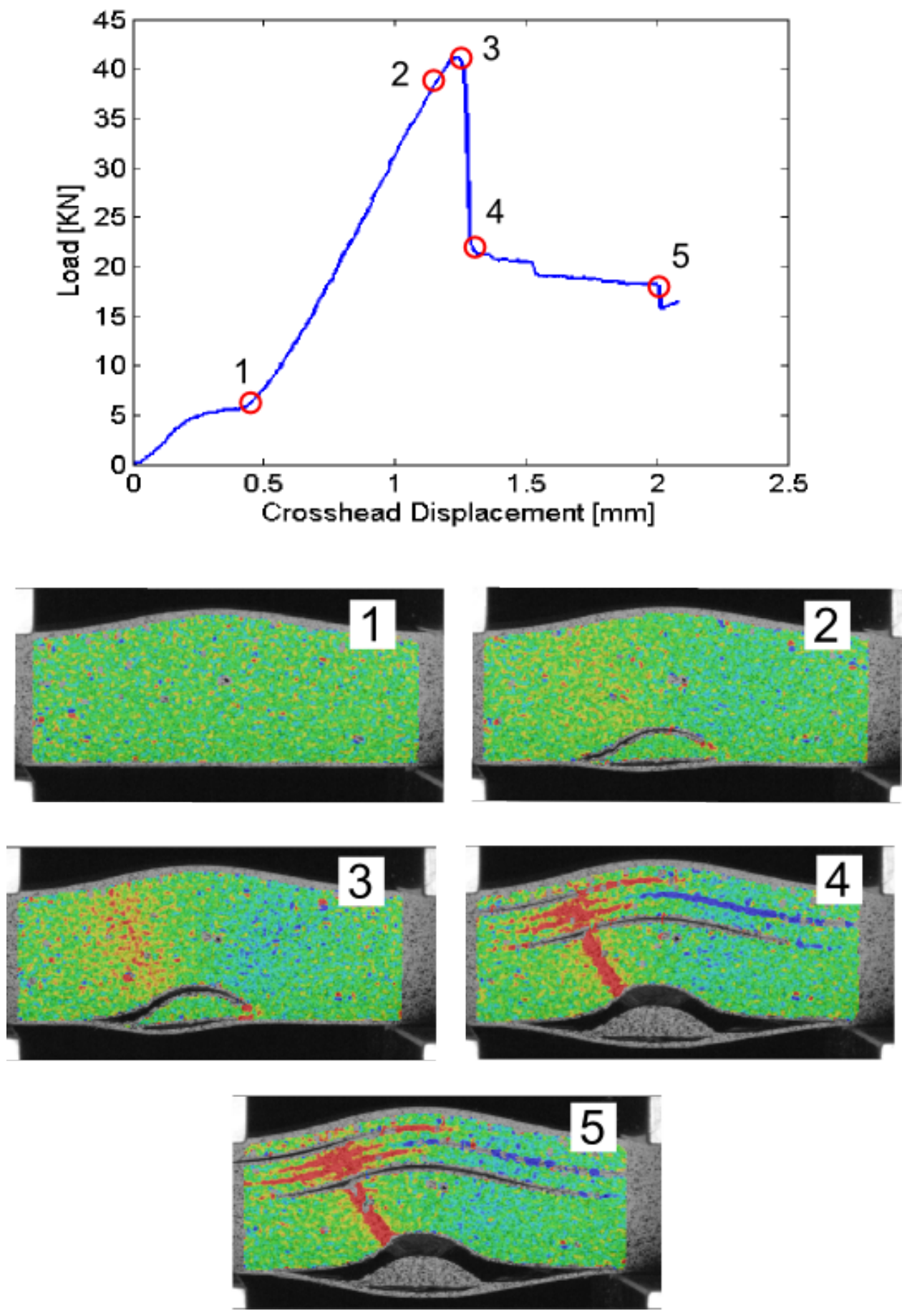

Figure 6. Load-displacement plot and corresponding shear strain DIC contours showing kink dominated failure subsequently leading to delamination. 

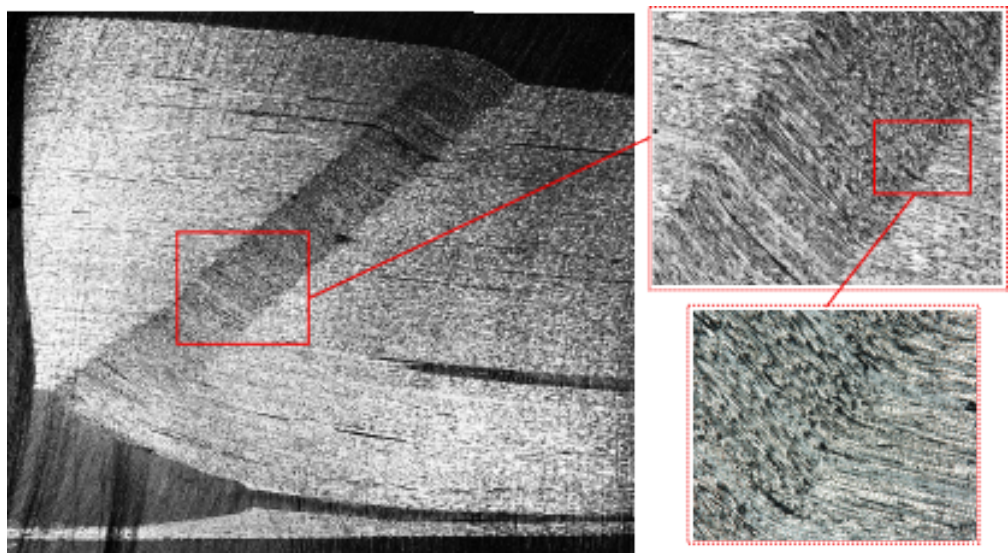

Figure 7. Micro-graphs showing kink band failure.

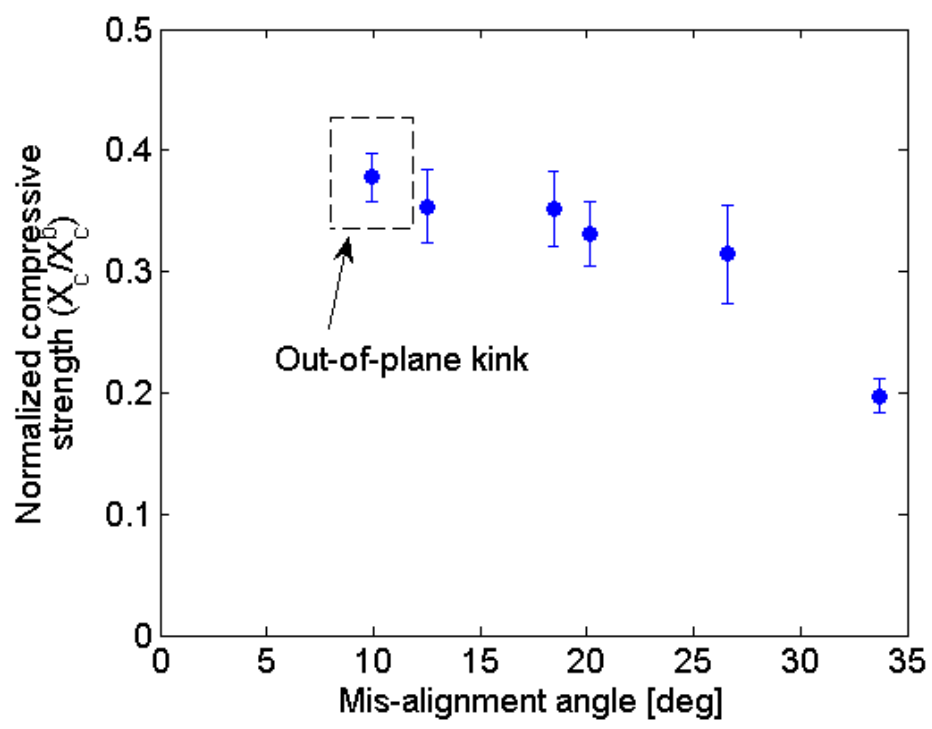

Figure 8. Normalized peak compressive stress plotted against misalignment angle $\alpha$ for inplane and out-of-plane kinking failure. 

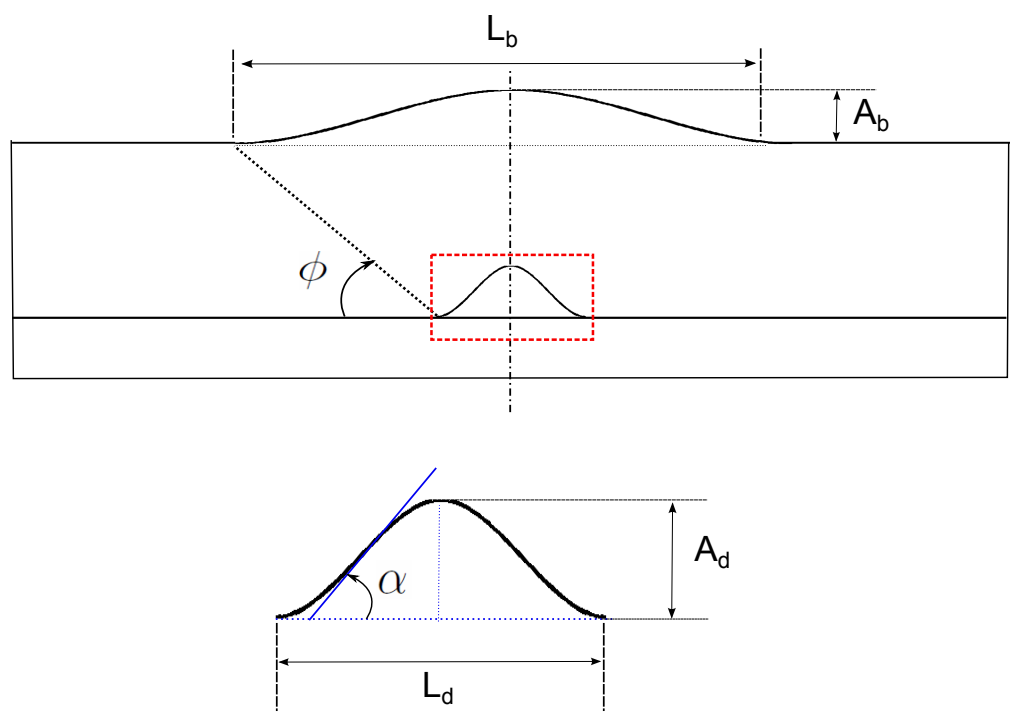

Figure 9. Geometry corresponding to the modeling parameters.

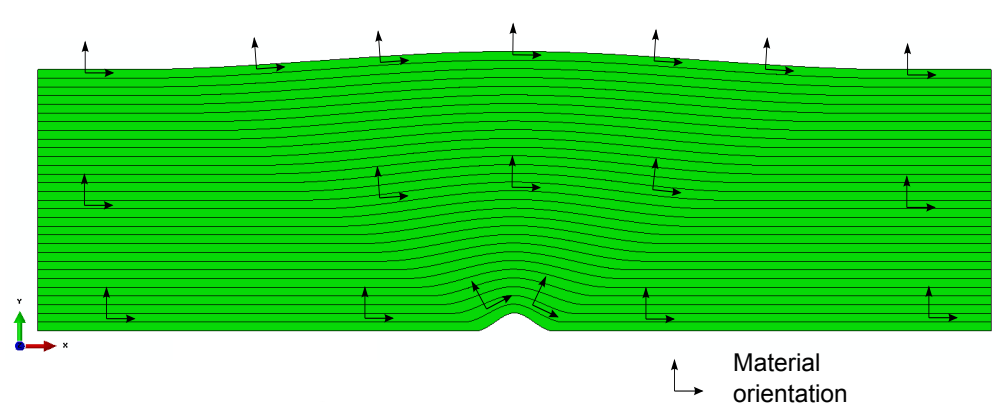

Figure 10. Modeling of layers and material orientation. 


\begin{tabular}{|c|}
\hline 1 \\
\hline Global macro \\
\hline Lamina-ortho \\
\hline (A-D) $u=0, v=0$ \\
(B-C) $u=-\delta, v=0$ \\
(A-B) $u=v=$ free \\
(C-D) $u=v=$ free \\
\hline Linear elastic \\
\hline
\end{tabular}

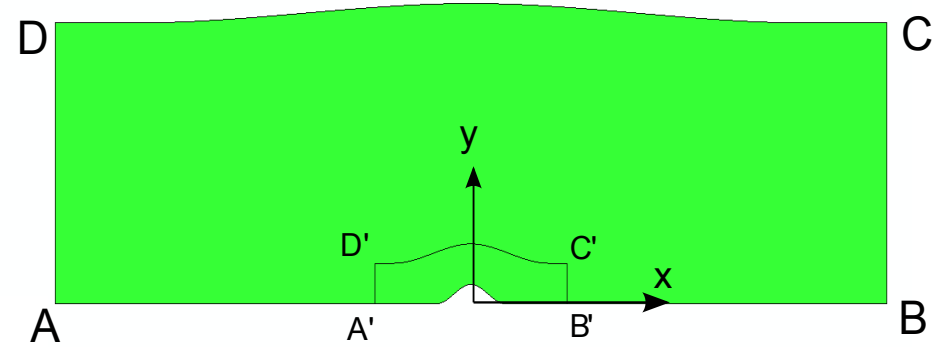

\begin{tabular}{|c|}
\hline 2 \\
\hline Local linear \\
\hline Fiber-orhto ; Matrix-iso \\
\hline (A'-D') $u=\Delta_{x}, v=\Delta_{y}$ \\
(B'-C') $u=\Delta_{x}, v=\Delta_{y}$ \\
(A'-B') $u=\Delta_{x}, v=\Delta_{y}$ \\
(C'-D') $u=\Delta_{x}, v=\Delta_{y}$ \\
\hline Linear elastic \\
\hline
\end{tabular}

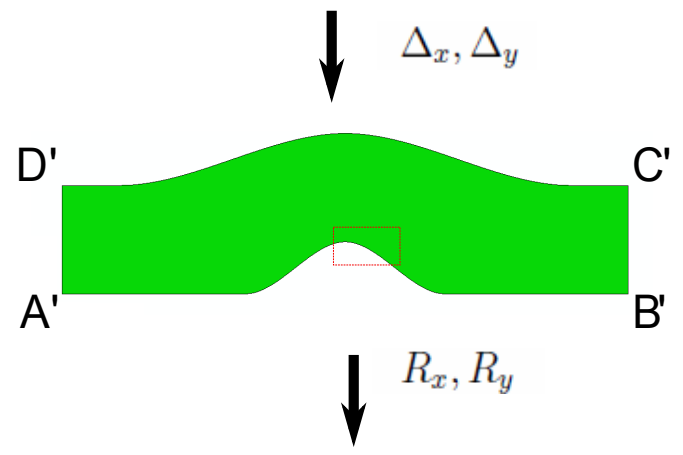

\begin{tabular}{|c|}
\hline 3 \\
\hline Local non-linear \\
\hline Fiber-orhto; Matrix-iso plastic \\
\hline (A'-D') $u=\Delta_{x}, v=\Delta_{y}$ \\
(B'-C') $u=\Delta_{x}, v=\Delta_{y}$ \\
(A'-B') $F_{x}=R_{x}, F_{y}=R_{y}$ \\
(C'-D') $F_{x}=R_{x}, F_{y}=R_{y}$ \\
\hline
\end{tabular}

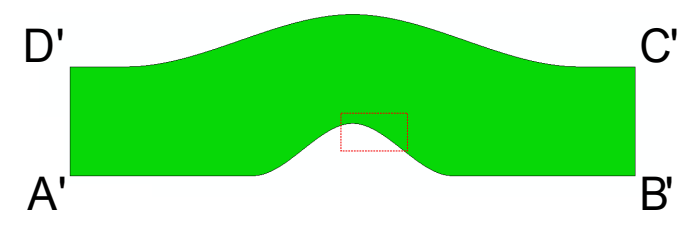

Fiber matrix mico-mechanics model

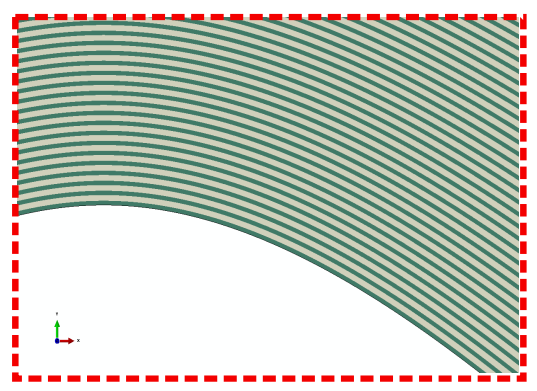

Figure 11. Global-local analysis procedure. 

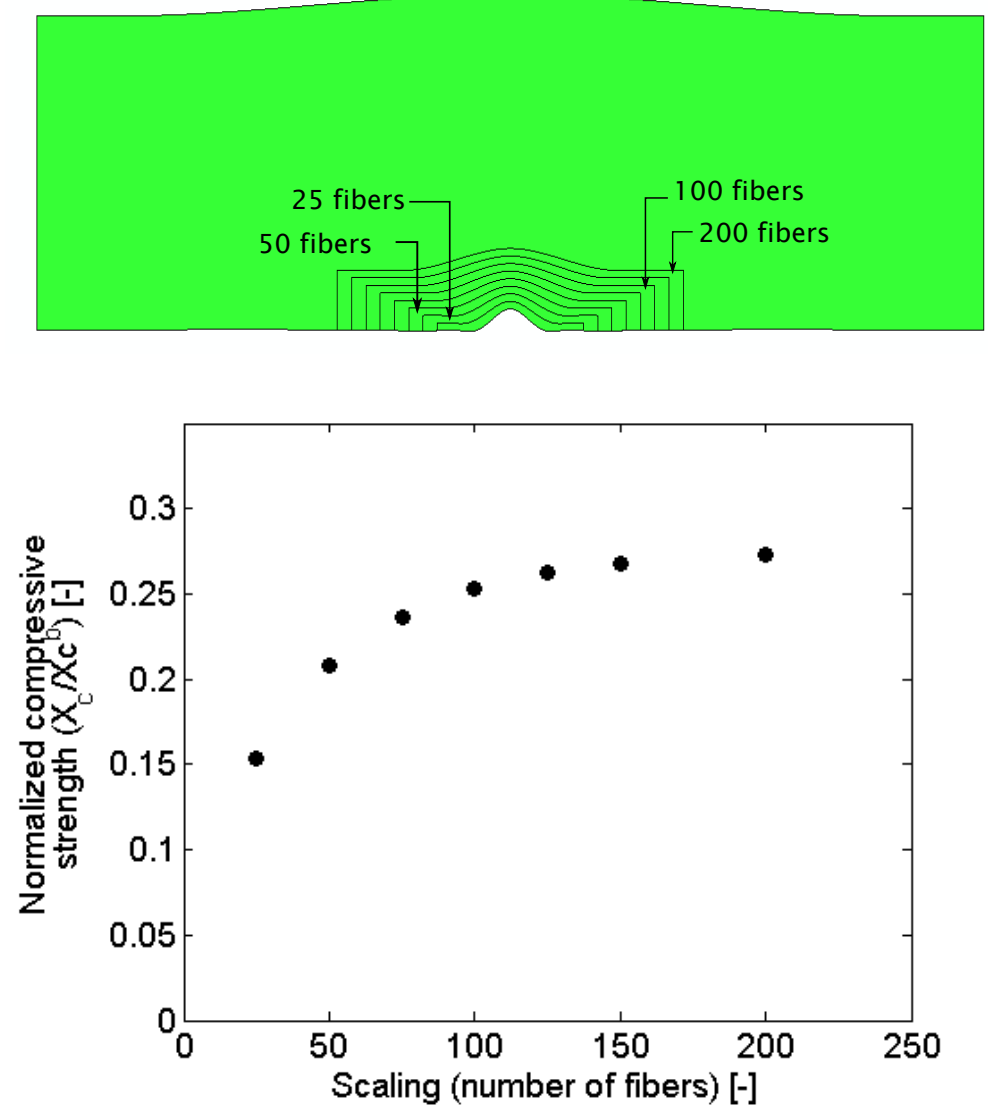

Figure 12. Scaling study to determine the local model size. 


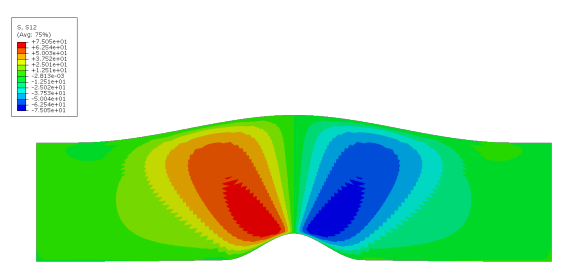

L. Step 1: Global model

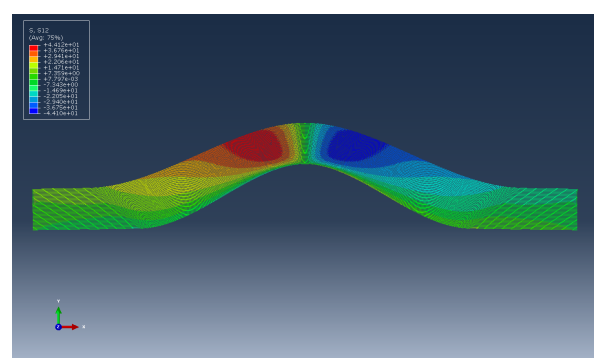

Step 2: Local model

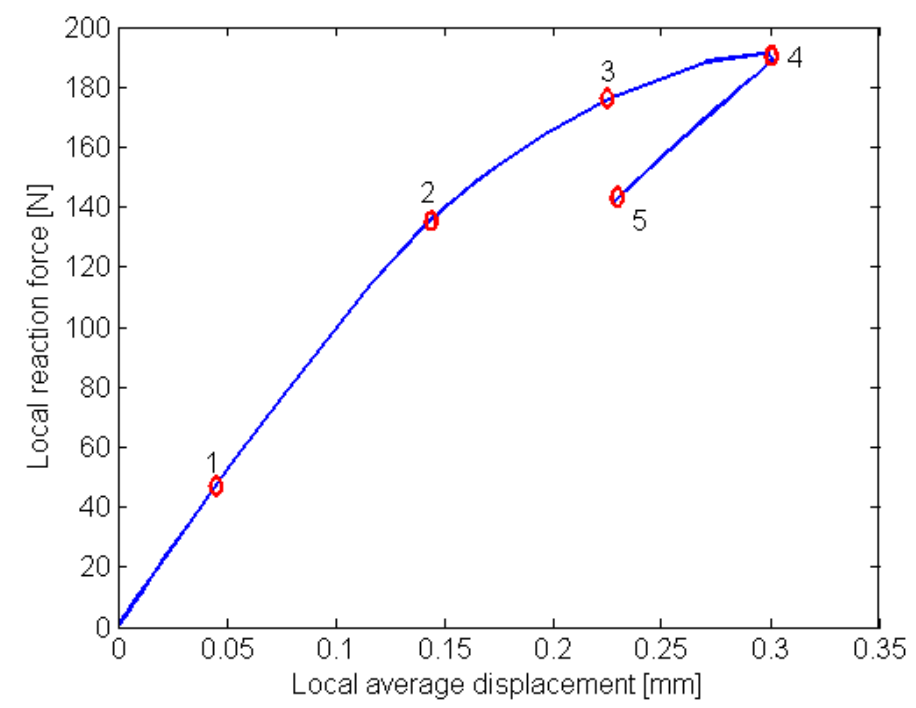

Local load displacement curve
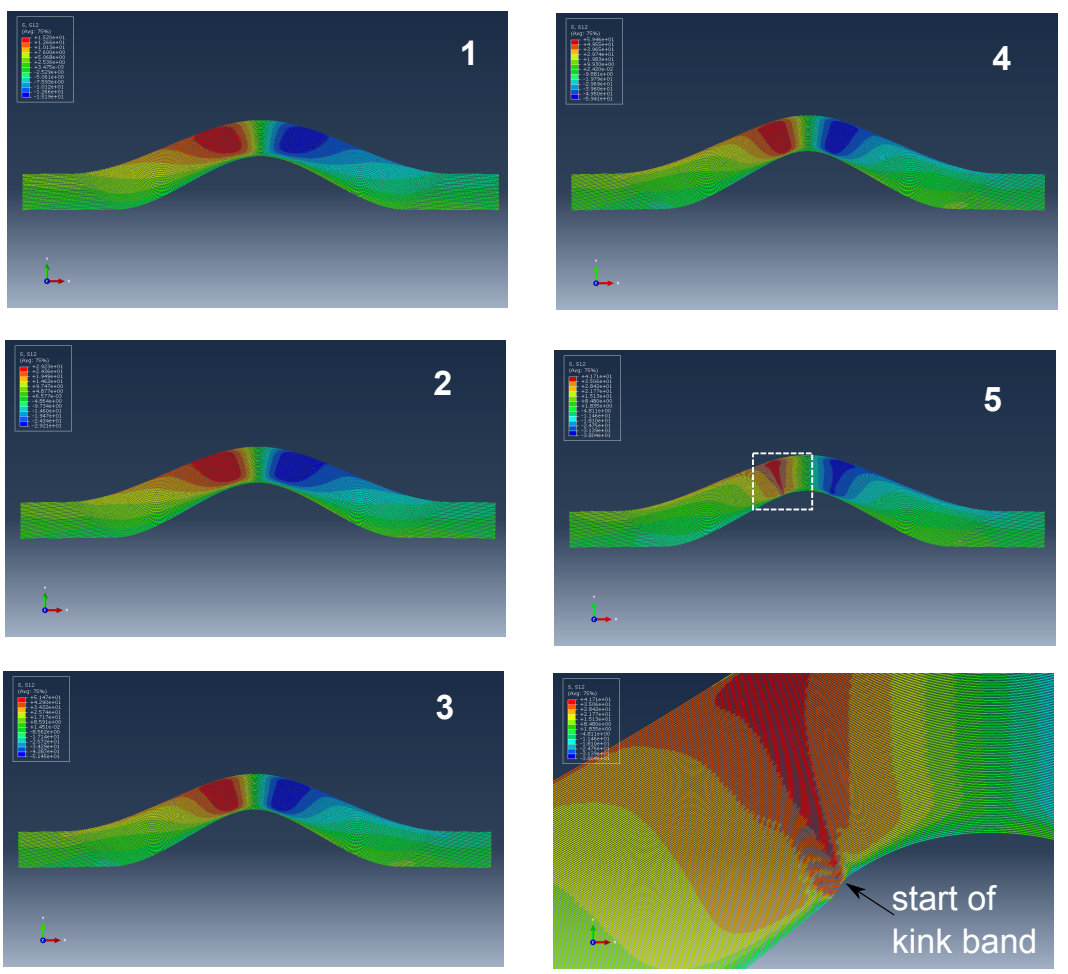

Step 3: Local non-linear model

Figure 13. Computational results from a global-local analysis with matrix shear stress plots showing evolution of shear stress and kink banding.

$$
15 \text { of } 16
$$




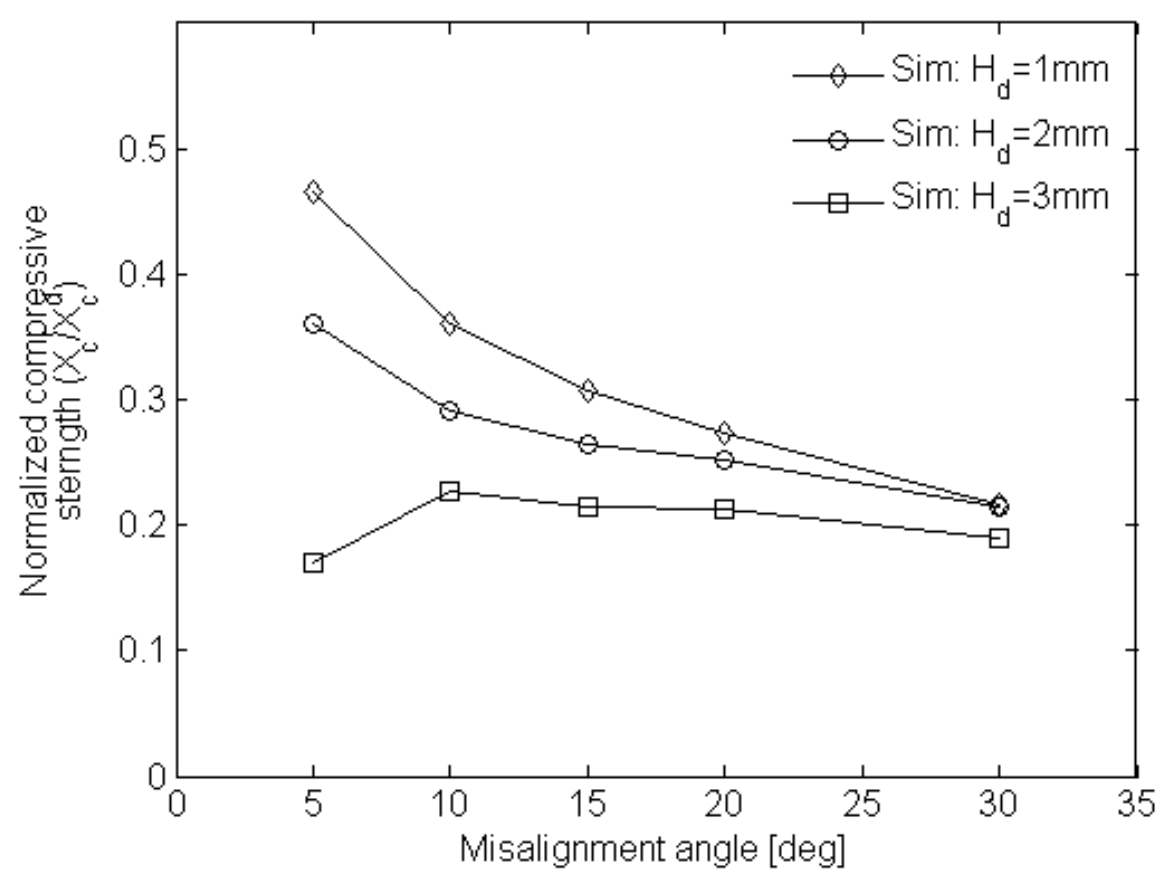

Figure 14. Parametric study results for in-plane kinking.

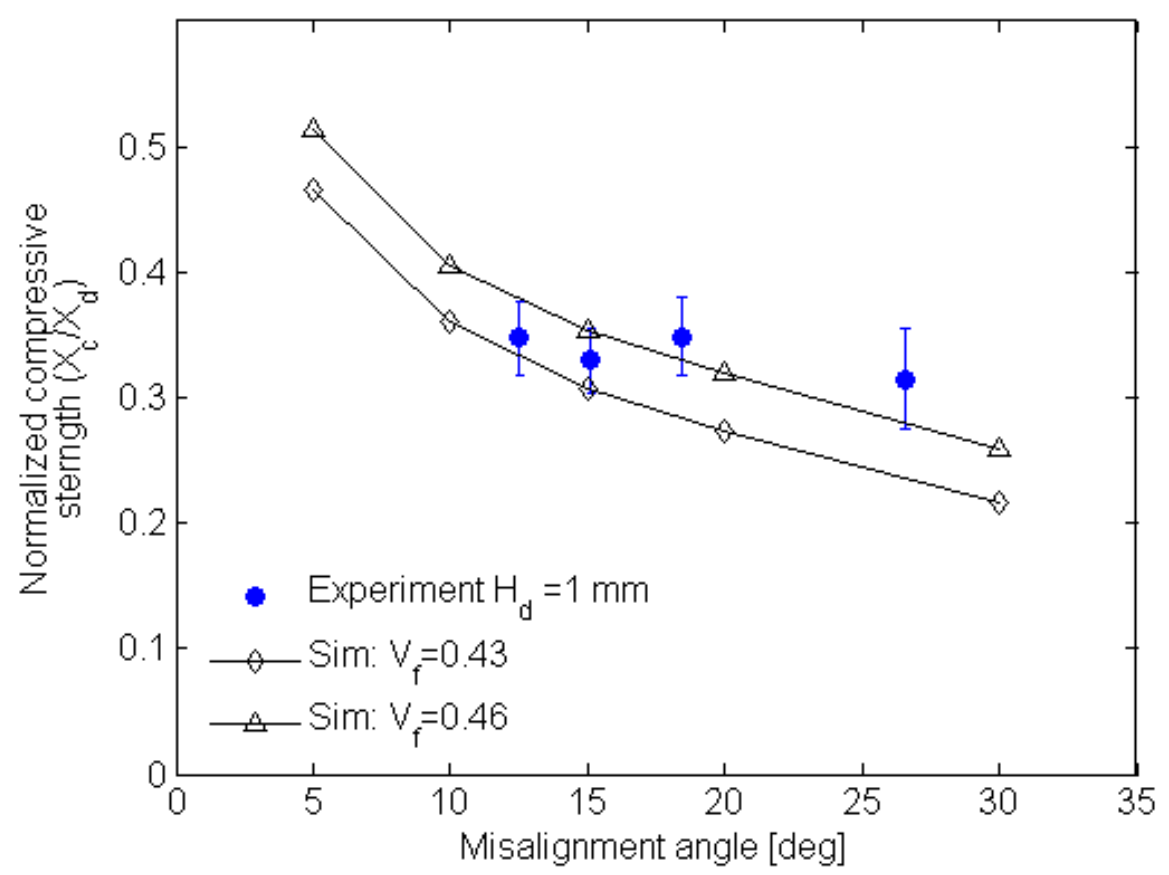

Figure 15. Micro-mechanical computational results compared against experimental results. 